\title{
Effect of HPT processing followed by long term natural ageing on mechanical and electrical properties of commercially pure $\mathrm{Cu}$
}

\author{
A. Rijal ${ }^{1}$, S.P. Singh ${ }^{1}$, J. K. Han ${ }^{2}$, M. Kawasaki², P. Kumar ${ }^{\dagger, 1}$ \\ †praveenk@iisc.ac.in \\ ${ }^{1}$ Department of Materials Engineering, Indian Institute of Science, Bangalore 560012, India
${ }^{2}$ School of Mechanical, Industrial and Manufacturing Engineering, Oregon State University, Corvallis, OR 97331, USA
}

Commercially pure $\mathrm{Cu}$ was processed through high-pressure torsion (HPT) up to a shear strain of 1000 and naturally aged for 1.75 years by keeping the samples under laboratory conditions. Hardness of the freshly processed samples monotonically increased with the HPT strain; however, the samples processed to a shear strain of 2 to 20 demonstrated a remarkable drop in the hardness values after the natural ageing. Interestingly, the natural ageing was not effective in changing the hardness of HPT processed samples strained up to very high shear strains. Electrical resistivity of the HPT processed samples after natural ageing showed a non-monotonous variation with the HPT strain, wherein it increased and then decreased and finally again started to increase with increasing shear strain. A discussion on the role of total length of boundaries, which was measured using electron back-scattered diffraction technique, and residual stresses, which was measured using X-ray diffraction, in determining hardness and resistivity is presented to qualitatively understand the origin of the non-monotonous variations of these two properties in the commercially pure Cu. It is suggested that besides residual stress, crystal defects, such as dislocations and vacancies, might also play important roles in determining the effect of HPT processing on resistivity of $\mathrm{Cu}$.

Keywords: commercially pure $\mathrm{Cu}$, hardness, high-pressure torsion, natural ageing, residual stress.

\section{Introduction}

High-pressure torsion (HPT) is a widely used severe plastic deformation (SPD) processing technique, wherein large plastic strain can be readily imposed on a bulk material [1] Since a very high compressive pressure (e.g., 2-6 GPa) is applied onto the disc-shaped sample while imposing the torsional strain, the samples do not show visible cracks or any other sign of structural damage even after several numbers of turns. This remarkable ability of HPT in imposing large strains in a sample makes it quite attractive. As an outcome of HPT processing, ultrafine-grained materials with excellent combination of electrical (e.g., low resistivity) and mechanical properties (e.g., enhanced hardness, yield strength, etc.) are obtained [2,3].

Due to the nature of the HPT processing, a heterogeneity in the imposed strain (as well as strain rate) is established along the radial direction from the disk center in the processed samples [1-5]. This graded strain field is, therefore, responsible for variation in microstructure in the HPT processed samples. The microstructural variation includes variation in the grain size, twin boundaries, low angle boundaries, dislocation density, etc. $[4,5]$. This variation in microstructure accordingly affects material properties, such as hardness, electrical conductivity, yield strength, etc., in the gradual fashion across the diameter of the samples $[2,3,6]$. Due to the existence of the graded microstructure and the imposition of very large mechanical strains during
HPT processing, the grain and dislocation substructures of the processed samples may become metastable, which lead to their evolution during post-processing storage, even at room temperature. Hence, effect of room temperature ageing, also known as natural ageing, on mechanical, electrical and other properties of materials processed through HPT is an interesting subject of research in the general area of SPD. Accordingly, this study focuses on evaluating effects of natural ageing on different properties of commercially pure $\mathrm{Cu}$, which is one of the materials that is used for applications where a diverse set of properties, such as yield as well as ultimate tensile strength, electrical conductivity, etc., is important. It should be noted that HPT processed $\mathrm{Cu}$ exhibits good mechanical properties without losing its electrical properties, especially in the as-processed condition $[7,8]$.

A few studies have reported effects of long-term natural ageing on HPT processed $\mathrm{Cu}$ samples $[6,9,10]$. In a recent article [6], it was reported that the HPT processed-naturally aged samples of $\mathrm{Cu}$ had an excellent combination of high hardness and high electrical conductivity (it should be noted that the previous study [6] showed the classic strengthconductivity paradox, i.e., the results did not show a simultaneous increase in both conductivity and strength due to HPT processing). Most of the reported studies investigating the effect of natural ageing on the hardness and electrical resistivity of $\mathrm{Cu}$ have focused on establishing a correlation between the observed properties and the microstructure, in terms of grain size, crystallographic texture, grain boundary 
misorientations, etc. However, it should also be noted that since HPT processing also induces a significant strain (about $1000)$ with strain gradient along radial direction while also applying large compressive pressure, the samples are expected to incur significant residual stress. Since the residual stress may affect both the mechanical behavior and the electrical (as well as thermal) transport, the latter by affecting the scattering cross-section of the atoms, it is imperative to study the effect of HPT processing on the residual stress in the samples. Accordingly, residual stresses in HPT processed commercially pure $\mathrm{Cu}$ after 1.75 years of natural ageing were measured using high resolution X-ray diffraction (XRD). The values of residual stresses obtained are then compared with the earlier reported hardness and resistivity of HPT processed $\mathrm{Cu}$ to gain insights into the importance of the microstructure and the residual stress on the observed trends.

\section{Materials and experimental procedure}

This study was conducted on commercially pure electrical grade $\mathrm{Cu}$. The details of HPT processing, sample preparation, hardness and resistivity measurements are discussed in detail in an earlier report [6] and thus only a brief description is provided in this report for continuity. Annealed $\mathrm{Cu}$ discs with $10 \mathrm{~mm}$ diameter and $0.85 \mathrm{~mm}$ thickness were subjected to $1 / 2,10$ and 50 turns of quasi-constraint HPT processing under a compressive pressure of $6 \mathrm{GPa}$ at room temperature, and subsequently stored under ambient conditions for 1.75 years. Samples were metallographically polished and their grain structure was analyzed using electron backscattered diffraction (EBSD). The main goals of the EBSD analysis were to obtain the grain size and measure the total length of the boundaries (including low as well as high angle grain boundaries and twin boundaries) as a function of the HPT shear strain. Vickers microhardness was measured along multiple radial directions in each HPT processed disc. In addition, small samples of approximately $1.5 \times 1.0 \times 0.55 \mathrm{~mm}^{3}$ size were carefully cut along the diameter of each disc for resistivity measurements using four probe method. It is important to note that all the measurements were conducted along the radial direction from the center of the $\mathrm{Cu}$ discs, as the strain varies radially in HPT processed discs.

The samples cut for resistivity measurements were utilized to evaluate the residual stresses using XRD. Prior to the XRDbased analysis, each small sample was mounted in an epoxy resin, which is an amorphous polymer. This mounting in the amorphous resin enabled fixing of such small samples on the X-ray diffractometer stage without interference or background from the Al stage of the diffractometer. A fourcircle high resolution X-ray diffractometer, with $0.015^{\circ}$ resolution, was utilized to conduct the $\mathrm{X}$-ray measurements to calculate the residual stresses. A step size of $0.03^{\circ}$ was utilized to collect the data in the diffractometer. It should be noted that due to the residual stress in a material, the original lattice spacing, $d_{0}$, changes and results in a different lattice spacing, $d$. Hence, to satisfy the Bragg's law for the new lattice spacing, the position of the XRD peak for a plane must shift. Hence, measurement of the peak shift, as compared to the unstrained sample, readily allows calculation of the residual stress. The maximum tensile or compressive residual stress, $\sigma_{\text {res }}$, is given by:

$$
\sigma_{r e s}=E \frac{\Delta 2 \theta}{4 \tan \theta}
$$

where $E$ is the elastic modulus of the material at the test temperature (which is equal to $128 \mathrm{GPa}$ for $\mathrm{Cu}$ at room temperature), $\Delta 2 \theta$ is the peak shift due to the lattice strain and $\tan \theta$ is calculated from the reference annealed samples peaks [11]. It should be noted that all measurements in this study were performed at room temperature. The combination of X-ray wavelength, incidence and diffracted angle gave a penetration depth of only $4.46 \mu \mathrm{m}$ and hence the measured residual stress is representative of top surface only. However, it should be noted that the existence of a residual stress in the top layer implies presence of residual stresses in the bulk of the sample also, for ensuring overall force balance in the sample.

\section{Results and Discussion}

\subsection{Measurement of residual stresses}

As mentioned in the previous section, the residual stress in the HPT processed-naturally aged $\mathrm{Cu}$ samples was evaluated by calculating the shift, relative to the unstrained condition, in the position of the $2 \theta$-peak corresponding to (111) plane using Eq. (1). This shift in the peak position was calculated relative to the reference peak for the annealed $\mathrm{Cu}$ samples where the annealed sample is considered to have no residual stress. Fig. 1a shows a representative $I-2 \theta$ plot showing a shift in the peak position for a $\mathrm{Cu}$ sample with an HPT strain of 22 relative to the peak of the annealed $\mathrm{Cu}$. A rightward shift in the peak position of (111) plane was readily discernable in Fig. 1a, which indicates a decrease in the interplanar spacing and hence it corresponds to a compressive residual stress. Although it appears reasonable, given the large compressive stress applied during HPT processing, it also indicates that there would be a tension-compression asymmetry in the mechanical properties of HPT processed-naturally aged $\mathrm{Cu}$ (e.g., difference in yield strength, etc.); this should be investigated further. Furthermore, as mentioned before, a fraction of the section will also be under tensile residual stress to maintain the force balance and hence evaluation of tension-compression asymmetry in HPT processed sample should be performed carefully.

Fig. $1 \mathrm{~b}$ shows the variation of the estimated residual stresses in the top layer of the HPT processed and naturally aged $\mathrm{Cu}$ samples. It should be noted that the strain shown on the abscissa in this graph corresponds to the mean of the shear strains across the sample investigated and the corresponding residual stress value shown on the ordinate is the average of three sets of readings obtained from the sample by mounting it on the sample holder at three different occasions. Since variation in the location of the top surface of the sample with respect to the source-detector position can result in a shift in the peak position, the above adopted method was applied to minimize the effect of positioning of the sample onto the sample-stage for the XRD measurements. In addition, a consistency between the top surface of the sample and the 
prescribed position of the sample for analysis was ensured by calibrating height adjustment each time before taking readings. As revealed by small error bars associated with all readings, positioning of the sample in the $\mathrm{X}$-ray diffractometer was repeatable and hence it is expected to be at the prescribed position. It can be observed from Fig. $1 \mathrm{~b}$ that the residual stress in all the HPT samples varied a little with strain and can be inferred to be within a band of $72 \pm 5 \mathrm{MPa}$ (in compression), which was attained just after a shear strain of 4 .

\subsection{Measurement of microhardness}

The variation of microhardness with strain is shown in Fig. 2 a. For comparison purposes, a dashed line showing the microhardness value of $48 \mathrm{HV}$ for the annealed $\mathrm{Cu}$ is also shown in Fig. 2 a. The plot readily reveals that for the as-fabricated sample (i.e., within $48 \mathrm{~h}$ of HPT processing), the microhardness increased to $145 \mathrm{HV}$ upon imposing an equivalent strain of 5 . After this increase, the hardness remained constant within a band of $145 \pm 5 \mathrm{HV}$ for strains up to 990 . On the other hand, the hardness of the samples processed under the same HPT processing conditions and subsequently naturally aged for 1.75 years shows nonmonotonous variation. The hardness decreased from $135 \mathrm{HV}$ to $80 \mathrm{HV}$ after an equivalent strain of 2 followed by ageing for 1.75 years, and thereafter it again increased with further increase in HPT strain (beyond a shear strain of $\sim 7-8$ ) and remained constant at $148 \pm 7 \mathrm{HV}$. The variation in the hardness of HPT processed-naturally aged sample with HPT strain was, hence, similar to that of the as-processed samples, at high strains (e.g., from 30 to up to 900). The deviation between the hardness of naturally aged and the as-fabricated samples is highlighted by a dashed rectangle in Fig. 2 a.

Fig. $2 \mathrm{~b}$ shows the variation of the hardness of the HPT processed and naturally aged $\mathrm{Cu}$ as a function of the inverse of the square root of its grain size, as dictated by the HallPetch relationship. Generally, an excellent match between the obtained data and the Hall-Petch relationship is obtained;

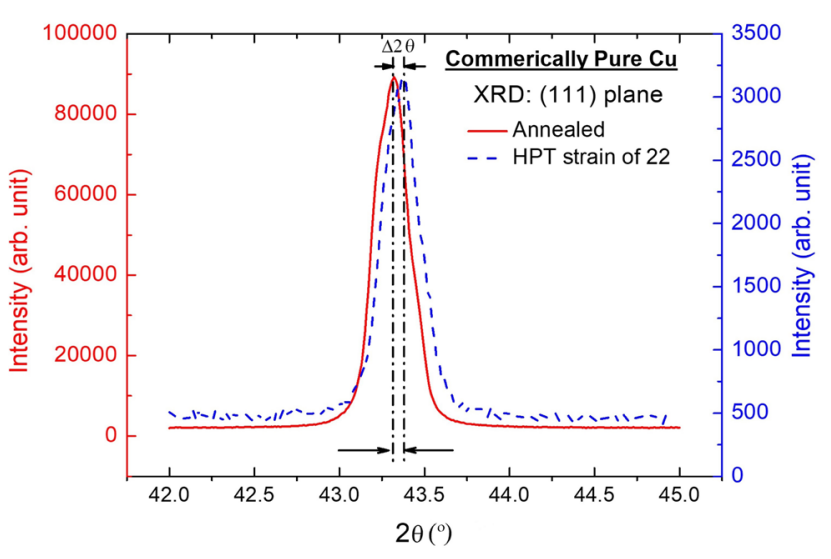

a

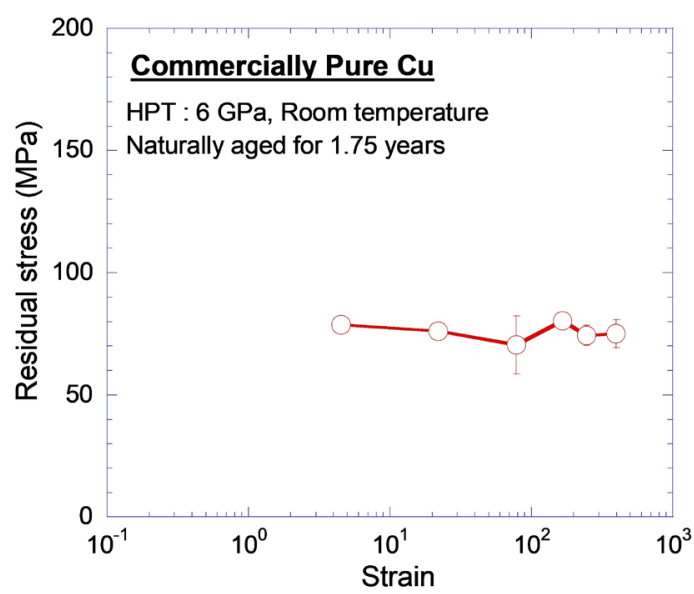

b

Fig. 1. (Color online) An example plot showing the shift in the peak position obtained in XRD pattern due to the presence of residual stresses (a), and variation of the residual stresses near the surface of the sample (up to a depth of $4.46 \mu \mathrm{m}$ ) with HPT strain in the samples processed through HPT followed by natural ageing for 1.75 years (b).

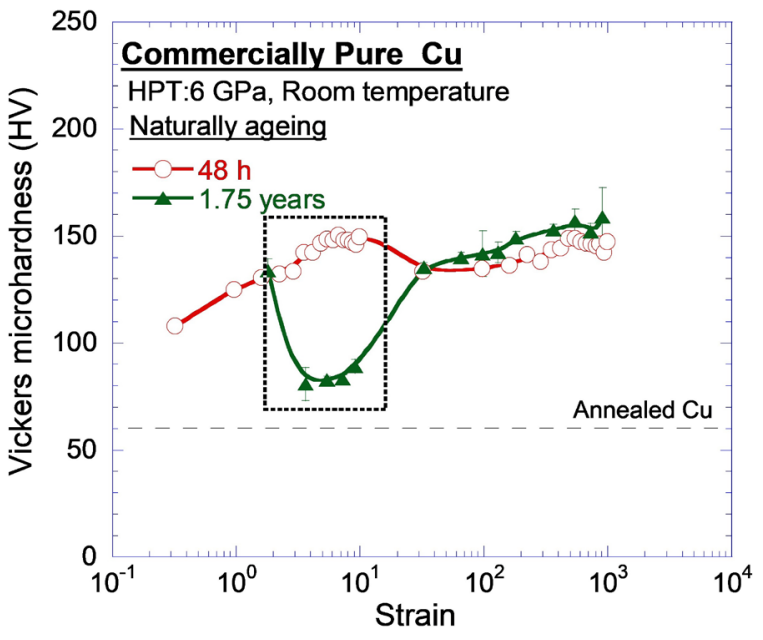

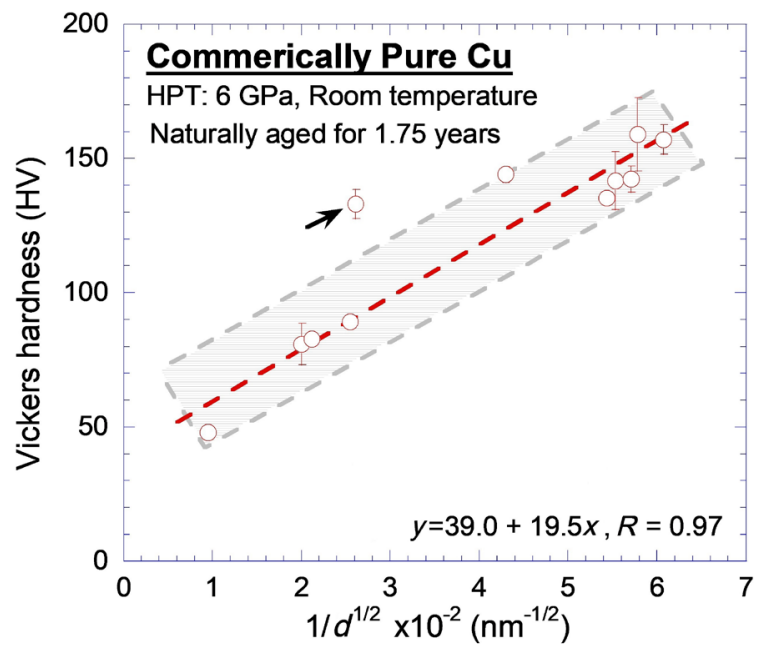

b

Fig. 2. Effect of HPT processing followed by natural ageing on hardness of commercially pure Cu: Variation of Vickers microhardness as function of the strain imposed during HPT (a) and inverse of the square root of grain size (b). The broken line shows the best fit curve, whose equation is given at the right-bottom corner of the graph. Here, the curve fitting line does not consider the "outlier" point shown by an arrow. The data shown in (b) has been taken from reference [6]. 
however, there is a notable exception which is shown by an arrow in Fig. 2 b. This point corresponds to the central region of the disc processed to $1 / 2$ turn of HPT. If this point is considered during curve fitting, a relatively poorer curve fitting regression is obtained (e.g., 0.97 if this datum point is excluded as compared to 0.90 if this datum point is also considered). Possible origins of this deviation may be the storage of a large fraction of sub-grains in this region and the variation in the residual stresses.

Yield stress, $\sigma_{\mathrm{YS}}$, was calculated from the Vickers microhardness value using the relation, $\sigma_{\mathrm{YS}} \approx(1 / 3) H_{\mathrm{HV}}$ where $H_{\mathrm{HV}}$ is the Vickers microhardness value calculated in units of $\mathrm{Pa}$ [12]. Fig. 3 a shows a comparative plot of yield stresses and the residual stress for the sample processed by HPT followed by natural ageing. It can be observed that the residual stresses generated by HPT processing did not have any significant effect on the hardness or yield stress values at high strains. Thus, the exceptionally high value of hardness at higher strains (including at a strain of less than 2, wherein it shows a high hardness value before the onset of the dip in the value - see Fig. 2 a) is not primarily due to the residual stresses present in the sample.

Another possible explanation for the deviation from the Hall-Petch relationship can be due to the role of the low angle grain boundaries (LAGB) in hardening of the material, especially when their fraction is high compared to high angle grain boundaries (HAGB). Accordingly, variation of the yield strength of HPT processed-naturally aged $\mathrm{Cu}$ samples was plotted against the total length of LAGB per unit area, which is shown in Fig. 3 b. A reasonably good linear fit between $\sigma_{\mathrm{YS}}$ and $l_{\mathrm{LAGB}}$ when the $l_{\mathrm{LAGB}}$ has not saturated explains the deviation of the particular datum point from the classical Hall-Petch relation at the low strains, as shown in Fig. 2 b. This can be attributed to the fact that classic Hall-Petch relation does not consider the strengthening due to the low angle grain boundaries. Based on this observation, it is suggested that the high hardness value at strain of 1 is possibly due to the high fraction of LAGB observed in the central region of the $1 / 2$ turn HPT processed sample (interestingly, this was shown to be true in an earlier publication [6]).

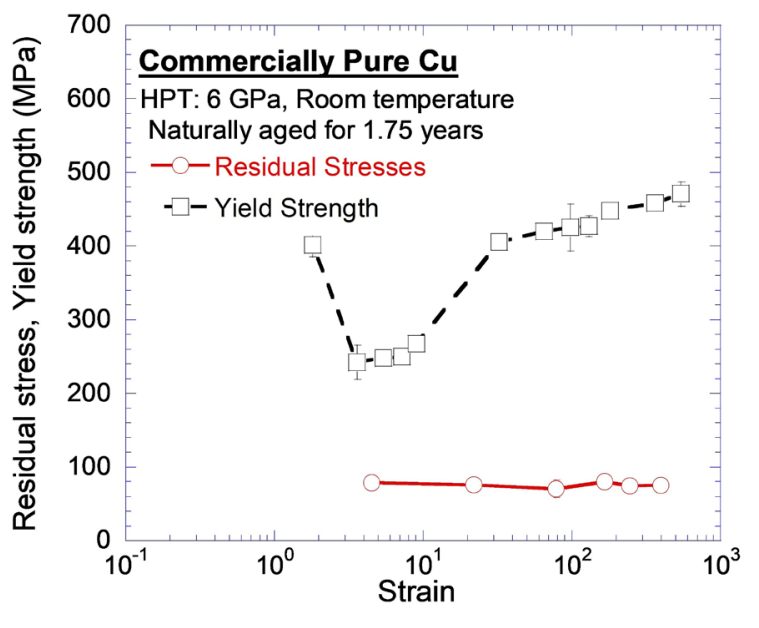

a

\subsection{Measurement of electrical resistivity}

Fig. 4 a shows the variation of the electrical resistivity of the HPT processed-naturally aged $\mathrm{Cu}$ samples as a function of the average shear strain imposed by HPT. The figure clearly shows that the electrical resistivity increased with HPT processing at lower strains and then it decreased with subsequent HPT straining, thereafter increased again to a relatively larger value at very high strains. The possible obstacles in the path of electrons in a material can be boundaries (such as grain boundaries, twin boundaries, etc.), defects (such as dislocations, vacancies, etc.), etc. At first, a correlation between the electrical resistivity and total boundary length was explored. Here, as shown in Fig. $4 \mathrm{~b}$, the total length of all types of boundaries increased monotonically up to a strain of $\sim 100$ and then it decreased to become saturated after a strain of $\sim 250$. This trend is opposite of that shown by the electrical resistivity (see Fig. 4 a) and hence the contribution of scattering of electrons by the boundaries was not significant in these samples. On the other hand, if the variation in the residual stress is observed (see Fig. 4b), then it appears that a decrease in the residual stress leads to a decrease in the electrical resistivity at low as well as moderate HPT strains (e.g., up to $\sim 10^{2}$ ). Since the residual stress suggests a change in the lattice spacing, it would imply a difference in the scattering efficiency of lattice points in the stressed lattice as compared to the unstressed lattice. However, a careful observation of Figs. $4 \mathrm{a}, \mathrm{b}$ reveals that at very high strains where residual stress becomes saturated (e.g., above $3 \times 10^{2}$ ), the electrical resistivity kept on increasing. Hence, it can be inferred that although the residual stress should be considered for understanding the variation of the electrical resistivity in $\mathrm{Cu}$ due to HPT straining, it may not be solely responsible for the observed variation. It is inferred that defect concentration, such as dislocation density and vacancy concentration (it is known that HPT processing leads to the creation of vacancies in excess to the expected equilibrium concentration of vacancies at the given temperature [13]), should also be considered and further explored to truly understand the

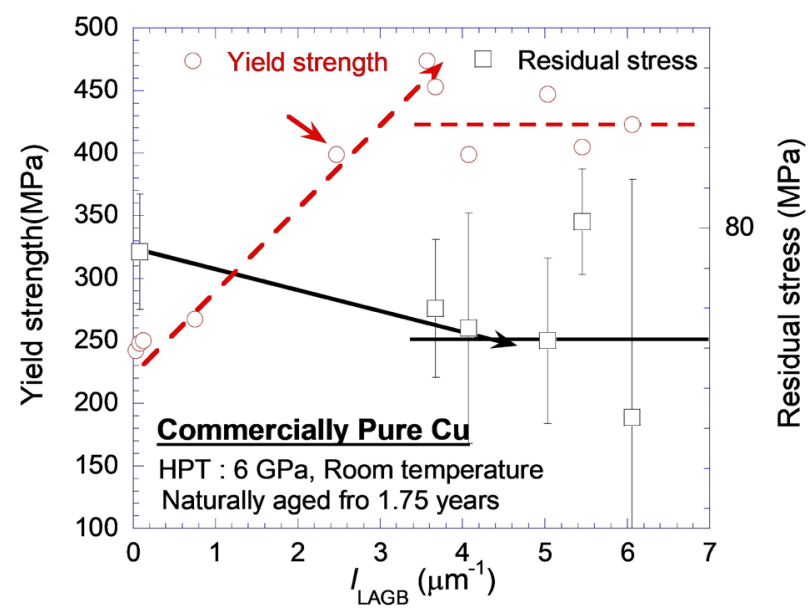

$\mathrm{b}$

Fig. 3. Variation of the yield strength of material (as calculated from Vickers microhardness data) and the residual stress as a function of HPT shear strain (a), and the yield stress as a function of total length of low-angle grain boundaries (LAGB) per unit area (b). 


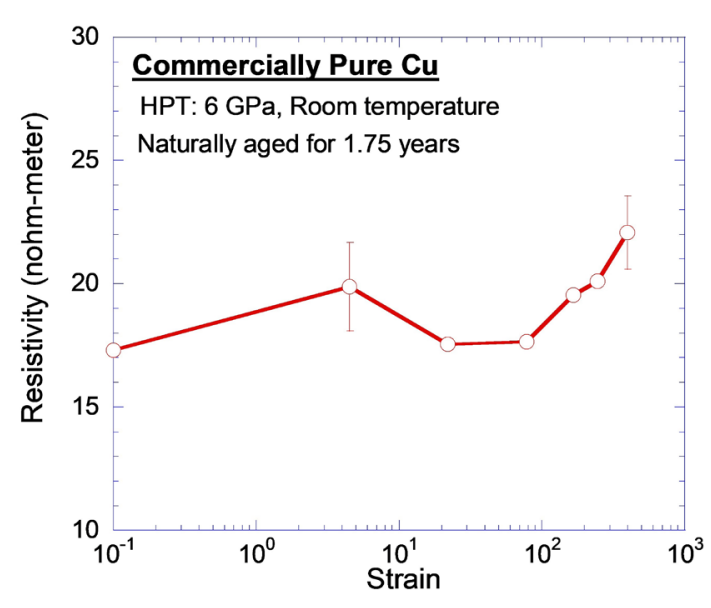

a

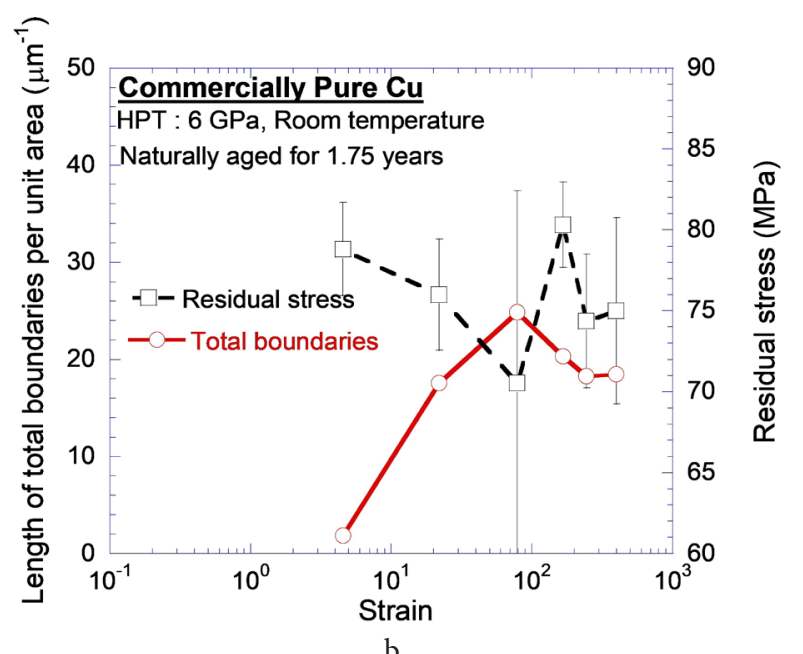

b

Fig. 4. Variation of electrical resistivity (a) and length of total grain boundaries per unit area as well as the residual stress as function of strain (b). The data shown in (a) have been taken from reference [6].

dominant route through which HPT straining affects the electrical resistivity of commercially pure $\mathrm{Cu}$.

\section{Conclusion}

A large amount of distortion in the material imposed by HPT can possibly generate significant residual stresses in the lattice. The value of the residual stress becomes saturated quickly, however with a little variation, with strain imposed by HPT.

It is possible that LAGB's are quite effective in enhancing the hardness of the HPT processed samples, especially at low strains. Hence, caution should be taken while plotting the Hall-Petch relationship for HPT processed samples, as it will be valid only when the fraction of LAGB's is neither significant nor evolving relative to the HAGB's.

The resistivity of the HPT processed-naturally aged samples varied non-monotonically with the HPT strain. While it is apparent that the residual stress affects the electrical resistivity of $\mathrm{Cu}$, especially at low strains, it is suggested that the role of defect concentration is further explored to truly understand the role of HPT processing in electrical resistivity.

Acknowledgements. This study was supported in part by Ministry of Human Resource Development, Government of India (AR, SPS and PK), Department of Science and Technology (DST), India through its National Clean Coal Research and Development Program (PK), and the National Science Foundation of the United States under Grant No. DMR1810343 ( $M K$ and JK).

\section{References}

1. J. Wongsa-Ngam, M. Kawasaki, T. G. Langdon. J. Mater. Sci. 48, 4653 (2013). Crossref

2. R.Z. Valiev, Y. Estrin, Z. Horita, T.G. Langdon, M. J. Zehetbauer, Y.T. Zhu. JOM. 58 (4), 33 (2000). Crossref

3. R.Z. Valiev, T.G. Langdon. Prog. Mater. Sci. 51, 881 (2006). Crossref

4. Y. Estrin, A. Molotnikov, C.H. Davies, R. Lapovok, J. Mech. Phys. Solids. 56, 1186 (2008). $\underline{\text { Crossref }}$

5. A.P. Zhilyaev, S. Lee, G. V. Nurislamova, R.Z. Valiev, T. G. Langdon. Scripta Mater. 44, 2753 (2001). Crossref

6. A. Rijal, S. P. Singh, J. K. Han, M. Kawasaki, P. Kumar. Adv.Eng. Mater. 1900547 (2019). Crossref

7. K. Edalati, K. Imamura, T. Kiss, Z. Horita. Mater. Trans. 53, 123 (2012). $\underline{\text { Crossref }}$

8. A.P. Zhilyaev, I. Shakhova, A. Belyakov, R. Kaibyshev, T. G. Langdon. J. Mater. Sci. 49, 2270 (2014). Crossref

9. Y. Huang, S. Sabbaghianrad, A.I. Almazrouee, K. J. Al-Fadhalah, S. N. Alhajeri, T. G. Langdon. Mater. Sci. Eng. A - Struct. 656, 55 (2016). Crossref

10. A.I. Almazrouee, K. J. Al-Fadhalah, S.N. Alhajeri, Y. Huang, T. G. Langdon. Adv. Eng. Mater. 21(5), 1801300 (2019). Crossref

11. B. D. Cullity, R.S. Stuart. Elements of X-ray Diffraction. 3rd ed. New Jersey, Prentice Hall (2001).

12. J.R. Cahoon, W.H. Broughton, A.R. Kutzak. Metall. Trans. 2, 1979 (1971).

13. M. Zehetbauer, G. Steiner, E. Schafler, A. V. Korznikov, E. Korznikova. Mater. Sci. Forum. 503, 57 (2006). Crossref 